\title{
Incidence, prevalence, and trajectories of repetitive conduction patterns in human atrial fibrillation
}

Citation for published version (APA):

van Rosmalen, F., Maesen, B., van Hunnik, A., Hermans, B. J. M., Bonizzi, P., Bidar, E., Nijs, J., Maessen, J. G., Verheule, S., Delhaas, T., Schotten, U., \& Zeemering, S. (2021). Incidence, prevalence, and trajectories of repetitive conduction patterns in human atrial fibrillation. EP Europace,

23(Supplement_1), i123-i132. https://doi.org/10.1093/europace/euaa403

Document status and date:

Published: 04/03/2021

DOI:

10.1093/europace/euaa403

Document Version:

Publisher's PDF, also known as Version of record

Document license:

Taverne

Please check the document version of this publication:

- A submitted manuscript is the version of the article upon submission and before peer-review. There can be important differences between the submitted version and the official published version of record.

People interested in the research are advised to contact the author for the final version of the publication, or visit the DOI to the publisher's website.

- The final author version and the galley proof are versions of the publication after peer review.

- The final published version features the final layout of the paper including the volume, issue and page numbers.

Link to publication

\footnotetext{
General rights rights.

- You may freely distribute the URL identifying the publication in the public portal. please follow below link for the End User Agreement:

www.umlib.nl/taverne-license

Take down policy

If you believe that this document breaches copyright please contact us at:

repository@maastrichtuniversity.nl

providing details and we will investigate your claim.
}

Copyright and moral rights for the publications made accessible in the public portal are retained by the authors and/or other copyright owners and it is a condition of accessing publications that users recognise and abide by the legal requirements associated with these

- Users may download and print one copy of any publication from the public portal for the purpose of private study or research.

- You may not further distribute the material or use it for any profit-making activity or commercial gain

If the publication is distributed under the terms of Article $25 \mathrm{fa}$ of the Dutch Copyright Act, indicated by the "Taverne" license above, 


\title{
Incidence, prevalence, and trajectories of repetitive conduction patterns in human atrial fibrillation
}

\section{Frank van Rosmalen', Bart Maesen $\mathbb{1}^{2}{ }^{2}$, Arne van Hunnik ${ }^{3}$, Ben J.M. Hermans ${ }^{3}$, Pietro Bonizzi ${ }^{4}$, Elham Bidar ${ }^{2}$, Jan Nijs $\mathbb{1}^{5}{ }^{5}$, Jos G Maessen ${ }^{2}$, Sander Verheule ${ }^{3}$, Tammo Delhaas $\mathbb{1}^{1}{ }^{1}$, Ulrich Schotten ${ }^{3 *}$, and Stef Zeemering ${ }^{3}$}

\begin{abstract}
${ }^{1}$ Department of Biomedical Engineering, Cardiovascular Research Institute Maastricht, Maastricht University, Maastricht, The Netherlands; ${ }^{2}$ Department of Cardiothoracic Surgery, Maastricht University, Medical Center \& Cardiovascular Research Institute Maastricht, Maastricht University, Maastricht, The Netherlands; ${ }^{3}$ Department of Physiology, Cardiovascular Research Institute Maastricht, Maastricht University, Maastricht, The Netherlands; ${ }^{4}$ Department of Data Science and Knowledge Engineering, Maastricht University, Maastricht, The Netherlands; and ${ }^{5}$ Department of Cardiac Surgery, UZ Brussel, Brussels, Belgium
\end{abstract}

Received 30 November 2020; editorial decision 2 December 2020; accepted after revision 11 December 2020

Aims

Repetitive conduction patterns in atrial fibrillation (AF) may reflect anatomical structures harbouring preferential conduction paths and indicate the presence of stationary sources for AF. Recently, we demonstrated a novel technique to detect repetitive patterns in high-density contact mapping of AF. As a first step towards repetitive pattern mapping to guide $\mathrm{AF}$ ablation, we determined the incidence, prevalence, and trajectories of repetitive conduction patterns in epicardial contact mapping of paroxysmal and persistent AF patients.

Methods and results

A 256-channel mapping array was used to record epicardial left and right AF electrograms in persistent AF (persAF, $n=9$ ) and paroxysmal AF (pAF, $n=11$ ) patients. Intervals containing repetitive conduction patterns were detected using recurrence plots. Activation movies, preferential conduction direction, and average activation sequence were used to characterize and classify conduction patterns. Repetitive patterns were identified in 33/40 recordings. Repetitive patterns were more prevalent in PAF compared with persAF [pAF: median 59\%, interquartile range (41-72) vs. persAF: 39\% (0-51), $P<0.01]$, larger [pAF: $=1.54(1.15-1.96)$ vs. persAF: $1.16(0.74$ 1.56) $\left.\mathrm{cm}^{2}, P<0.001\right)$, and more stable [normalized preferentiality (0-1) pAF: $0.38(0.25-0.50)$ vs. persAF: $0.23(0$ $0.33), P<0.01]$. Most repetitive patterns were peripheral waves (87\%), often with conduction block (69\%), while breakthroughs $(9 \%)$ and re-entries $(2 \%)$ occurred less frequently.

Conclusion High-density epicardial contact mapping in AF patients reveals frequent repetitive conduction patterns. In persistent AF patients, repetitive patterns were less frequent, smaller, and more variable than in paroxysmal AF patients. Future research should elucidate whether these patterns can help in finding AF ablation targets.

Keywords Atrial fibrillation • Repetitive patterns • Conduction patterns • High-density epicardial • atrial mapping

\section{Introduction}

Pulmonary vein isolation (PVI) is a common treatment for atrial fibrillation $(\mathrm{AF}) .^{1,2}$ In patients with paroxysmal AF, PVI leads to freedom of $A F$ in $59-89 \%$ of patients 12 months after the procedure. ${ }^{3}$ In patients with persistent AF only $59 \%$ show freedom of AF after 18 months. Atrial fibrillation recurrence after PVI can be caused by reconnection of pulmonary veins $(P V s)$, but recurrence of $A F$ is also possible while all $P V s$ are still isolated, implying the existence of $A F$ sources outside of the PVs. ${ }^{4}$ To improve the success rate of PVI, the identification of alternative ablation targets outside of the PVs has been explored. Ablation of dominant frequency sites, linear lesion sets, complex fractionated atrial electrograms (CFAE) as well as focal impulse, and rotor modulation ablation showed significant

* Corresponding author. Tel: +31433881200, E-mail address: schotten@maastrichtuniversity.nl

Published on behalf of the European Society of Cardiology. All rights reserved. (c) The Author(s) 2021. For permissions, please email: journals.permissions@oup.com. 


\section{What's new?}

- Repetitive conduction patterns during AF were detected in high-density contact mapping in patients, using recurrence plots.

- Repetitive conduction patterns were found in a majority of patients.

- Persistent AF patients show less frequent, smaller, and more variable conduction patterns than paroxysmal AF patients.

- The majority of repetitive conduction patterns was (blocked) waves entering from outside the mapping array.

- Repetitive breakthrough and re-entry patterns were observed in a small minority of cases.

- The existence of repetitive patterns during human AF suggests the presence of stationary AF drivers. Ablation of these drivers may restore sinus rhythm.

improvement in single studies, but in larger trials or meta-analyses their superiority as compared with PVI-only could not consistently be demonstrated. ${ }^{3,5-8}$

Repetitive focal sources ${ }^{9}$ and micro-re-entrant sources ${ }^{10,11}$ have been described as candidate sources of AF. Repetitive conduction patterns are expected to occur close to AF drivers, independent of their actual mechanism (local re-entry, ectopic firing, complex conduction with new wave generation). Detection of repetitive conduction patterns may help to localize sources of $A F$, and thus provide a new strategy for AF driver identification. Repetitive conduction patterns are also of interest because they might reflect anatomical structures harbouring preferential conduction paths associated with the underlying bundle structure of the atrium. ${ }^{10,12,13}$ Therefore, a mapping technique able to find repetitive patterns may help study structure-function relationships during fibrillatory conduction. Finally, the analysis of stable repetitive patterns may also facilitate combining recordings at different sites in the atria to reconstruct a composite activation map during $A F$, so far only feasible during stable activation patterns like atrial flutter or atrial tachycardia. These composite AF activation maps could help to increase the spatial coverage of existing mapping modalities. ${ }^{14}$

A technique to detect repetitive patterns in high-density mapping of AF has recently been demonstrated by our group. ${ }^{15}$ In this study, we applied this methodology to investigate the incidence, prevalence, and trajectories of repetitive conduction patterns in epicardial mapping data of paroxysmal and persistent AF patients.

\section{Methods}

\section{Patient selection}

This study uses a retrospective dataset of 20 patients [persistent AF (persAF), $n=9$ and paroxysmal $\mathrm{AF}(\mathrm{pAF}), n=11$ ] that underwent bi-atrial epicardial mapping. ${ }^{16}$ Patients included in this study were referred for open-chest surgery: either cut-and-sew rhythm surgery, coronary bypass grafting, aortic valve surgery, or mitral valve surgery. The study protocol was approved by the local ethics committee and informed consent was obtained from each patient before the procedure.

\section{Epicardial mapping procedure}

The heart was approached via median sternotomy. Two electrode grids were sequentially placed on the atria, one on the free wall of the right atrium (RA) and one in the oblique sinus on the posterior left atrium (LA). Both electrode grids were custom-made, single-use 256-channel grids measuring $22.5 \mathrm{~mm} \times 22.5 \mathrm{~mm}$ with an inter-electrode distance of $1.5 \mathrm{~mm}$. A reference electrode was positioned subcutaneously in the sternotomy opening. In pAF patients in sinus rhythm at the start of the procedure, AF was induced by incremental pacing $(300-60 \mathrm{~ms}$ cycle length). Recording of unipolar electrograms was started $30 \mathrm{~s}$ after $A F$ induction. For each location (RA and LA), 10 s of data was stored using a $1039 \mathrm{kHz}$ sampling rate.

\section{Electrogram processing}

The automated processing and annotation of AF electrograms have been described previously. ${ }^{17}$ Briefly, all electrograms were high-pass filtered using a third-order Chebyshev filter with a cut-off frequency of $0.5 \mathrm{~Hz}$ to remove baseline drift. Ventricular waves visible in the atrial recordings were detected and removed using single beat QRST-template cancellation. Local activations, characterized by sharp deflections on the electrogram, were detected using unipolar deflection template matching and classified as local activations using a probabilistic algorithm based on the estimated distribution of the AF cycle length (AFCL).

\section{Recurrence plot construction}

The methodology used to detect intervals containing repetitive patterns is described in detail in a recent study by Zeemering et al. ${ }^{15}$ Unipolar electrograms were transformed to an activation phase signal by treating the local activation time as the moment of phase inversion and using linear interpolation for the time between the phase inversions. This procedure resulted in a signal that represents the phase of the activity in a local electrogram, with values between $-\pi$ and $\pi$ and allowed for the creation of snapshots that represented the phase of all individual electrodes at one point in time (see Figure 1A). Snapshots at two different points in time were compared by computing the phase difference for every corresponding electrode between the two snapshots. The distance $\delta_{i, j}$ between two activation-phase snapshots at time points $i$ and $j$ was determined based on the phase angle difference at each of the $N$ electrodes, by taking the average of the cosine of each difference, transformed back to a fraction of the activation-phase duration of a single AF cycle $(2 \pi)$. Formally:

$$
\delta_{i, j}=\cos ^{-1}\left(\sum_{k=1}^{N} \cos \left(\varphi_{k, j}-\varphi_{k, i}\right) / N\right) / 2 \pi,
$$

where $\varphi_{k, i}$ denotes the activation-phase of the electrode $k$ at time $i$. This definition led to distance values between 0 (two snapshots completely in phase) and 0.5 (two snapshots half an AF cycle length out of phase). We used a recurrence plot to visualize whenever a pair of snapshots exhibited an activation-phase distance below a certain distance threshold. Recurrence plots show 'time' on both the $x$ - and $y$-axis. Snapshots with a distance smaller than a threshold are recurrences, represented by a dot (see Figure $1 B$ and $C$ ). As an example, a snapshot at $t=1 \mathrm{~s}$ that recurs at $t=5 \mathrm{~s}$ is shown in the recurrence plot by a dot at position $(1,5)$ as well as a dot at position $(5,1)$, since all recurrence plots are symmetric with respect to the diagonal. The threshold for the maximum-allowed activation-phase distance between two snapshots was computed based on the maximum expected number of recurrences. The maximum expected number of recurrences was estimated as the number of recurrences that would occur if a single conduction pattern repeatedly occurred for the full length of the recording, giving rise to on average one recurrence per 


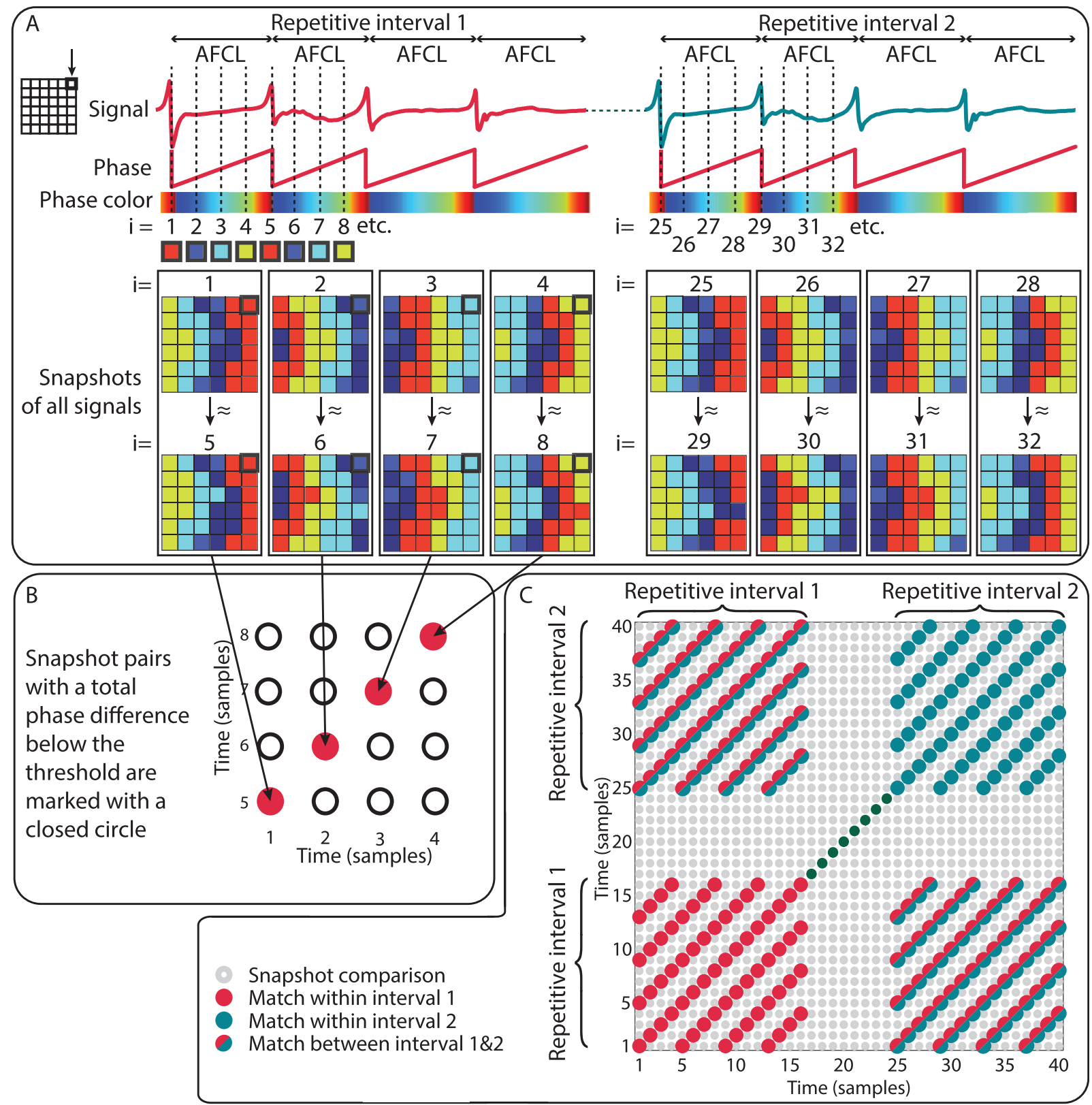

Figure I The creation of recurrence plots. (A) The signal for one electrode of a mapping grid. The signals consist of two repetitive intervals. The first repetitive interval (red trace) is repeated later in time (blue trace). The signal is converted to a phase signal (straight lines under signal trace), and snapshots consisting of the phase of all electrodes of the grid created. Snapshots are compared, and when the phase difference is below the phase difference threshold, the time points of similar phase snapshots are marked on a recurrence plot $(B)$. (C) The recurrence plot for the trace in panel $A$.

AF cycle length for each snapshot. This number therefore depended on the AF cycle length and the sampling frequency of the recording, since a higher sampling frequency results in more snapshots per time. The choice of the distance threshold enabled a sensitive detection of recurrences, which ensured that completely regular patterns with on average one recurrence per AF cycle length were detected correctly. In recordings with a lower degree of regularity, this choice of recurrence plot threshold caused false positive recurrence detections, which were removed in the next stage as explained below.

\section{Repetitive pattern detection}

A recurrence plot was generated for every recording, and used to detect the number and duration of repetitive patterns. A conduction pattern that is repetitive for a number of consecutive AF cycles has recurrences with a period of one AF cycle length. In the time interval that contains the repetitive pattern, every snapshot has recurrences with preceding or following snapshots, again with a period of one AF cycle length. This is visible in the recurrence plot as a block of stacked diagonal lines around the main diagonal with a distance of one AF cycle length between the 
diagonal lines (Figure 1C). Diagonal lines may exhibit some 'thickness' when the distance between consecutive activation-phase snapshots still falls within the computed distance threshold, which leads to aforementioned false positive recurrences. This effect was removed by erosion: replacing consecutive horizontal or vertical recurrences by a single recurrence at the pair of snapshots with minimum distance.

The eroded recurrence plot was used to detect intervals that contained successive repetitive conduction patterns. These intervals were detected by an algorithm that traversed the main diagonal of a recurrence plot and computed the recurrence rate in square blocks of increasing duration around the diagonal at each time point. The recurrence rate is the 'density' of a recurrence plot and is computed as the number of detected recurrences divided by all comparisons possible. Square blocks centred on the diagonal with a minimum recurrence rate of 0.9 (corresponding to an average of 0.9 recurrences per AF cycle) were selected as recurrent intervals.

\section{Characterization of repetitive conduction patterns}

We computed the preferential (dominant) conduction direction per individual electrode of each repetitive conduction pattern (circular variance of conduction direction, defined as Pref). ${ }^{13}$ A Pref of 1 indicates that all conduction vectors point in the same direction, whereas a Pref of 0 implies that all conduction vectors are uniformly pointing in all directions. Within repetitive intervals, the degree of preferentiality and direction of conduction for the mapping array were visualized in preferentiality plots, where arrows indicate the preferential direction, and Pref is indicated by a colour. The preferentiality plot only shows arrows for electrodes with a Pref $>0.5$, to highlight preferential conduction directions that could be assessed accurately. The size of a repetitive pattern was defined as the number of electrodes with Pref $>0.5$. The preferentiality of conduction direction during a repetitive pattern was defined as the sum of Pref over electrodes with a Pref $>0.5$.

Detected repetitive patterns may vary in duration and pattern stability. To compare Pref between intervals, we defined normalized preferentiality $\left(\right.$ Pref $\left._{\text {norm }}\right)$ as an overall measure of AF pattern stability. Pref $_{\text {norm }}$ incorporates pattern duration, size of the high preferential region, and the sum of Pref in the high preferential region into one metric. The duration of the interval is normalized to the total recording duration, the size of the high preferential region is normalized to the total size of the mapping array, and the sum of Pref in the high preferential region is normalized to the sum of the maximum Pref value for every electrode. Pref $f_{\text {norm }}$ is the product of the normalized duration, normalized high preferentiality region, and normalized sum of Pref, and thus has a value between 0 (short/small area/unstable pattern) and 1 (long/large area/stable pattern; see Supplementary material online). This implies that intervals in which all electrodes in the mapping array are part of the repetitive pattern, and where the pattern continues uninterrupted for the total duration of the recording, are assigned a value of one. In contrast, the normalized preferentiality is zero either when no interval containing a repetitive pattern can be found in the recording or no electrodes with a preferentiality $>0.5$ are present in the repetitive intervals.

The preferentiality plot, average activation sequence plot, and an animation of the activation pattern for each repetitive pattern were generated and visually inspected by three authors (F.R., S.Z., and U.S.). Patterns were categorized as peripheral wave without block, peripheral wave with block, breakthrough, re-entry within the mapping area, or undecided if the pattern did not fit any of the other categories. Definitions for conduction patterns are presented in Supplementary material online.

\section{Statistics}

Differences between pAF and persAF patients were analysed for the number of intervals containing repetitive patterns, the duration of these intervals, the size of the region with Pref $>0.5$, the sum of Pref $>0.5$, and

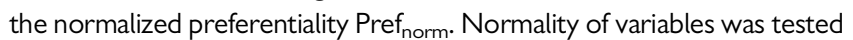
using the one-sample Kolmogorov-Smirnov test. To test for significant differences between AF groups, a mixed ANOVA was used, employing a significance threshold of 0.05 . Multiple comparison tests were performed using Tukey's range test, controlling for AF group and atrium. Descriptive statistics of variables with a normal distribution are reported as mean and standard deviation (SD). Descriptive statistics of other variables are indicated by median, 25th, and 75th percentile.

\section{Results}

Baseline characteristics of all patients are given in Table 1. In persAF patients, atrial size was larger, left atrial appendage flow velocity was lower, and $\mathrm{CHA}_{2} \mathrm{DS}_{2}-\mathrm{VASc}$ score and $\mathrm{ACE}$-inhibitors use were higher.

Measurements of all 20 patients were used, resulting in 20 recordings of the $L A$ and 20 recordings of the RA. Recurrent intervals were found in 33 out of 40 recordings (pAF: 20/22, persAF: 13/18, $P=0.12$ ). Although the incidence of recurrent intervals did not significantly differ between the paroxysmal and persistent AF group [PAF: median $=3$, inter-quartile range $(2-3)$ vs. persAF: median $=2$, $(0-4)$, $P=0.29$, Figure 2], the prevalence, defined as total repetitive pattern duration as percentage of total recording time, was higher in $\mathrm{PAF}$ patients [pAF: median $=59 \%(41-72)$ vs. persAF: median $=39 \%(0-$ 51), $P<0.01]$. The relation between the number of intervals and the total duration of intervals in a recording is visualized in Figure 2, bottom right. The size of repetitive patterns was significantly larger in PAF patients [pAF: median $=1.54(1.15-1.96)$ vs. persAF: median $=$ $1.16(0.74-1.56) \mathrm{cm}^{2}, P<0.001$, Figure 2, top right]. Preferentiality of conduction direction during a repetitive pattern was higher in paroxysmal than in persistent AF [PAF: median sum of preferentiality over all electrodes $=114$ (77-146), persAF: median sum of preferentiality over all electrodes $=82(67-114), P<0.01]$. Overall conduction pattern stability was significantly higher in $\mathrm{PAF}$ patients [PAF: median = $0.38(0.25-0.50)$ vs. persAF: median $=0.23(0-0.33), P<0.005$, Figure 2, bottom centre].

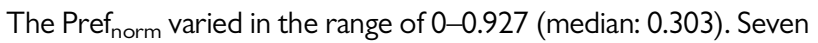
recordings showed a Pref norm $_{\text {of } 0 \text {, the lowest non-zero Pref }}$ norm was 0.090 . Figure 3 provides examples for left atrial recordings of a recurrence plot, preferentiality plot, and average activation sequence for a high (0.927), moderate (0.315), and low (0.104) non-zero normalized preferentiality.

Repetitive patterns were categorized based on their prevailing conduction trajectories. Classification results are shown in Figure 4. In right and left atria together, most repetitive patterns were peripheral waves $(87 \%)$, often with conduction block (69\%), while breakthroughs (9\%), and re-entries (2\%) occurred less frequently. Both paroxysmal and persistent AF showed more blocked waves than unblocked waves (pAF: $55 \%$ vs. $27 \%$, persAF: $92 \%$ vs. $3 \%$ ), but blocked waves were more common in persistent AF patients (PAF: 54\%, persAF: 92\%). Nine intervals showed a repeating pattern of breakthroughs and peripheral waves (pAF: 11\%, persAF: 5\%). 
Table I Patient characteristics

\begin{tabular}{|c|c|c|}
\hline & Paroxysmal AF $(n=11)$ & Persistent AF $(n=9)$ \\
\hline Age (year) & $68.7 \pm 4.2$ & $69.1 \pm 5.8$ \\
\hline Male (\%) & 75.0 & 55.6 \\
\hline \multicolumn{3}{|l|}{ Clinical characteristics } \\
\hline Nicotine abuse (\%) & 27.3 & 44.4 \\
\hline Hypertension (\%) & 63.6 & 77.8 \\
\hline Diabetes (\%) & 9.1 & 44.4 \\
\hline Peripheral arterial disease (\%) & 18.2 & 22.2 \\
\hline Previous MI (\%) & 18.2 & 11.1 \\
\hline Hypercholesterolaemia (\%) & 36.4 & 44.4 \\
\hline $\mathrm{GFR}<60 \mathrm{~mL} / \mathrm{min}(\%)$ & 18.2 & 22.22 \\
\hline $\mathrm{BMI}\left(\mathrm{kg} / \mathrm{m}^{2}\right)$ & $26.6 \pm 4.4$ & $26.9 \pm 3.1$ \\
\hline NYHA class & $2[1.25-2.75]$ & $2[2-3]$ \\
\hline $\mathrm{CHA}_{2} \mathrm{DS}_{2}$-VASc & $3[2-3]$ & $3[3-6]^{*}$ \\
\hline Time since AF diagnosis (year) & $7.0 \pm 6.9$ & $9.6 \pm 6.6$ \\
\hline Preoperative CRP (mg/L) & 2.3. \pm 1.5 & $3.2 \pm 3.7$ \\
\hline \multicolumn{3}{|l|}{ Surgery } \\
\hline CABG & 7 & 4 \\
\hline MVS & 1 & 2 \\
\hline AVS & 2 & 2 \\
\hline Lone AF & 1 & 1 \\
\hline \multicolumn{3}{|l|}{ Echocardiography } \\
\hline LA diameter $(\mathrm{mm})$ & $43.5 \pm 8.1$ & $53.1 \pm 6.1 *$ \\
\hline LA volume $\left(\mathrm{cm}^{3}\right)$ & $79.1 \pm 27.5$ & $151.0 \pm 55.3^{*}$ \\
\hline LAA flow velocity $(\mathrm{cm} / \mathrm{s})$ & $61.1 \pm 25.9$ & $37.6 \pm 13.3^{*}$ \\
\hline RA volume $\left(\mathrm{cm}^{3}\right)$ & $53.6 \pm 26.1$ & $102.8 \pm 32.3^{*}$ \\
\hline LVEDD (mm) & $52.0 \pm 4.4$ & $52.1 \pm 5.1$ \\
\hline LVESD (mm) & $32.8 \pm 6.0$ & $34.9 \pm 6.8$ \\
\hline LVEF (\%) & $64.3 \pm 9.1$ & $61.4 \pm 6.4$ \\
\hline \multicolumn{3}{|l|}{ Drug use } \\
\hline$\beta$-Blocker (\%) & 81.8 & 77.8 \\
\hline Digoxin (\%) & 0.0 & 33.3 \\
\hline Amiodarone/sotalol (\%) & 63.6 & 11.1 \\
\hline ACE-inhibitor/ARB (\%) & 54.5 & $100 *$ \\
\hline Calcium channel blocker (\%) & 27.3 & 33.3 \\
\hline
\end{tabular}

ACE, angiotensin converting enzyme; ARB, angiotensin-receptor blockers; AVS, aortic valve surgery; BMI, body mass index; CABG, coronary bypass grafting; CRP, c-reactive protein; GFR, glomerular filtration rate; LA, left atrial; LAA, left atrial appendage; LVEDD, left ventricular end diastolic diameter; LVEF, left ventricular ejection fraction; LVESD, left ventricular end systolic diameter; MI, myocardial infarction; MVS, mitral valve surgery; NYHA, New York Heart Association; RA, right atrial. $* P<0.05$ vs. paroxysmal AF.

\section{Discussion}

We investigated the presence, duration, area, and the trajectories of repetitive conduction patterns in epicardial high-resolution direct contact mapping data of patients with paroxysmal and persistent AF. Repetitive conduction patterns were identified in the majority of patients, both in PAF and persAF. Not only the prevalence of repetitive patterns was higher in $\mathrm{pAF}$ patients compared with persAF patients but also the overall stability of AF patterns. The majority of repetitive patterns was peripheral waves, often with conduction block, while breakthroughs and re-entries occurred less frequently.

\section{Atrial fibrillation pattern stability and atrial fibrillation complexity}

Analysis of recurrent patterns through normalized preferentiality showed that persAF patients exhibit patterns that are more variable compared with PAF patients. These findings may reflect well described changes of the atrial substrate under the influence of $A F$, where prolonged AF leads to more complex atrial substrate with a higher degree of block and a higher degree of electrical dissociation. ${ }^{18}$ This complex substrate may cause repeating AF patterns to become less stable in time and location. 

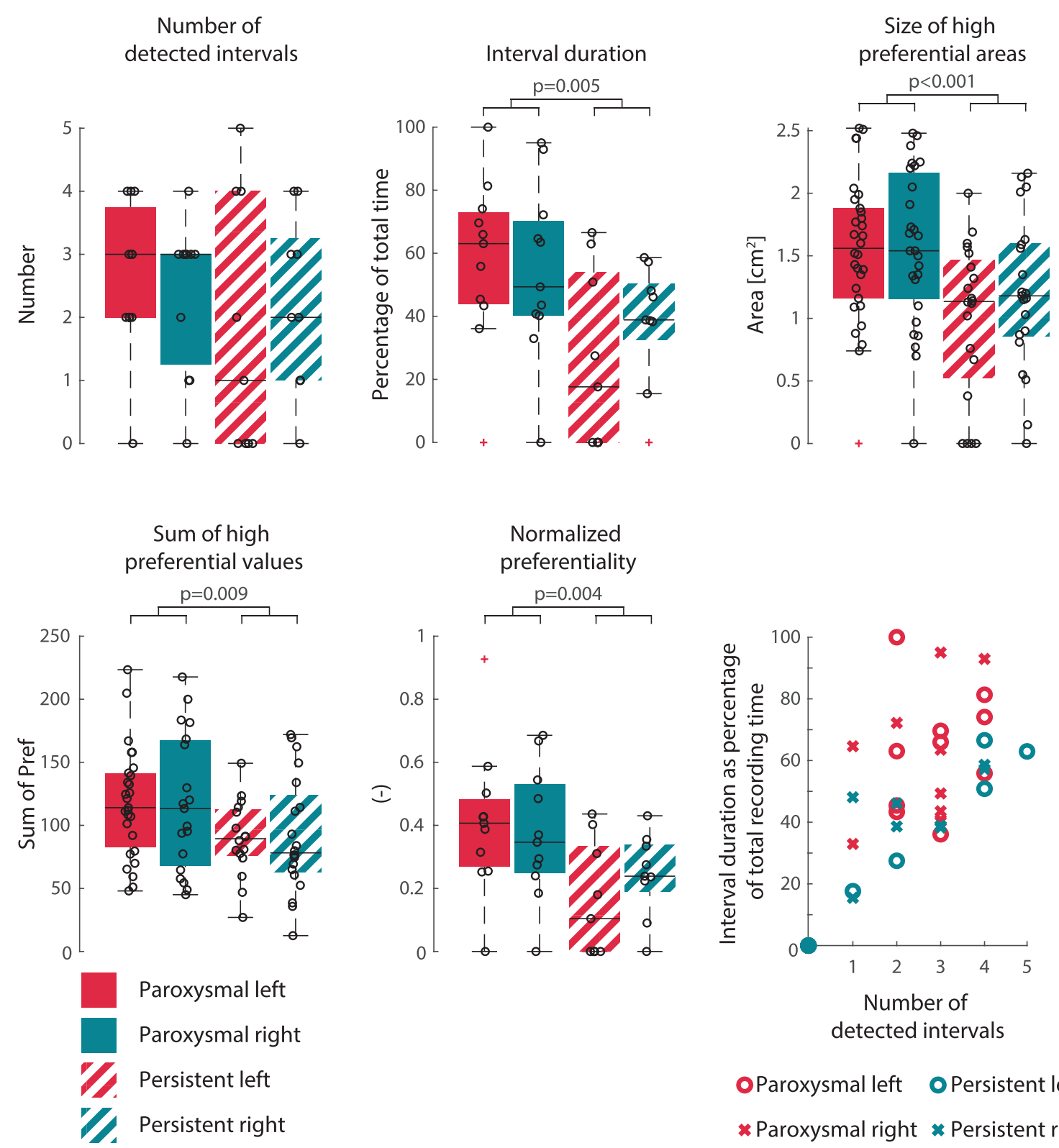

\section{OParoxysmal left OPersistent left \\ * Paroxysmal right $*$ Persistent right}

Figure 2 Summary of repetitive interval detection results. There was no significant difference in number of detected intervals between pAF and persAF. Interval duration as percentage of total time, size of high preferential areas, sum of high preferential values as well as the normalized preferentiality show a significant difference between pAF and persAF. pAF, paroxysmal atrial fibrillation; persAF, persistent atrial fibrillation.

\section{Classification of repetitive patterns}

In the present study, five types of repetitive conduction patterns were used: Peripheral with block, peripheral without block, breakthrough, re-entry, and undecided. Both PAF and persAF patients showed more blocked than unblocked waves, but blocked waves were more common in persAF. Nine intervals showed a repeating pattern of breakthroughs and peripheral waves. Re-entries were only found in two intervals.

Classification of the repetitive patterns revealed that the ratio of blocked vs. unblocked peripheral waves is higher in the persistent AF group. Uninterrupted peripheral waves were mostly observed in
PAF patients, but were almost absent in persAF patients. This indicates that patients with persistent $A F$ have a more complex atrial substrate with a higher incidence of conduction block. Interestingly, nine repetitive patterns were classified as breakthroughs. Repetitive breakthroughs, visible as radial spread of conduction from one point in the mapping array, may represent rapid ectopic firing or endoepicardial dissociation, and might be responsible for the perpetuation of AF. $9,19-22$

Peripheral waves without conduction block were found relatively more often for paroxysmal AF patients and may indicate a zone of unidirectional conduction that can be part of a macro-re-entry 


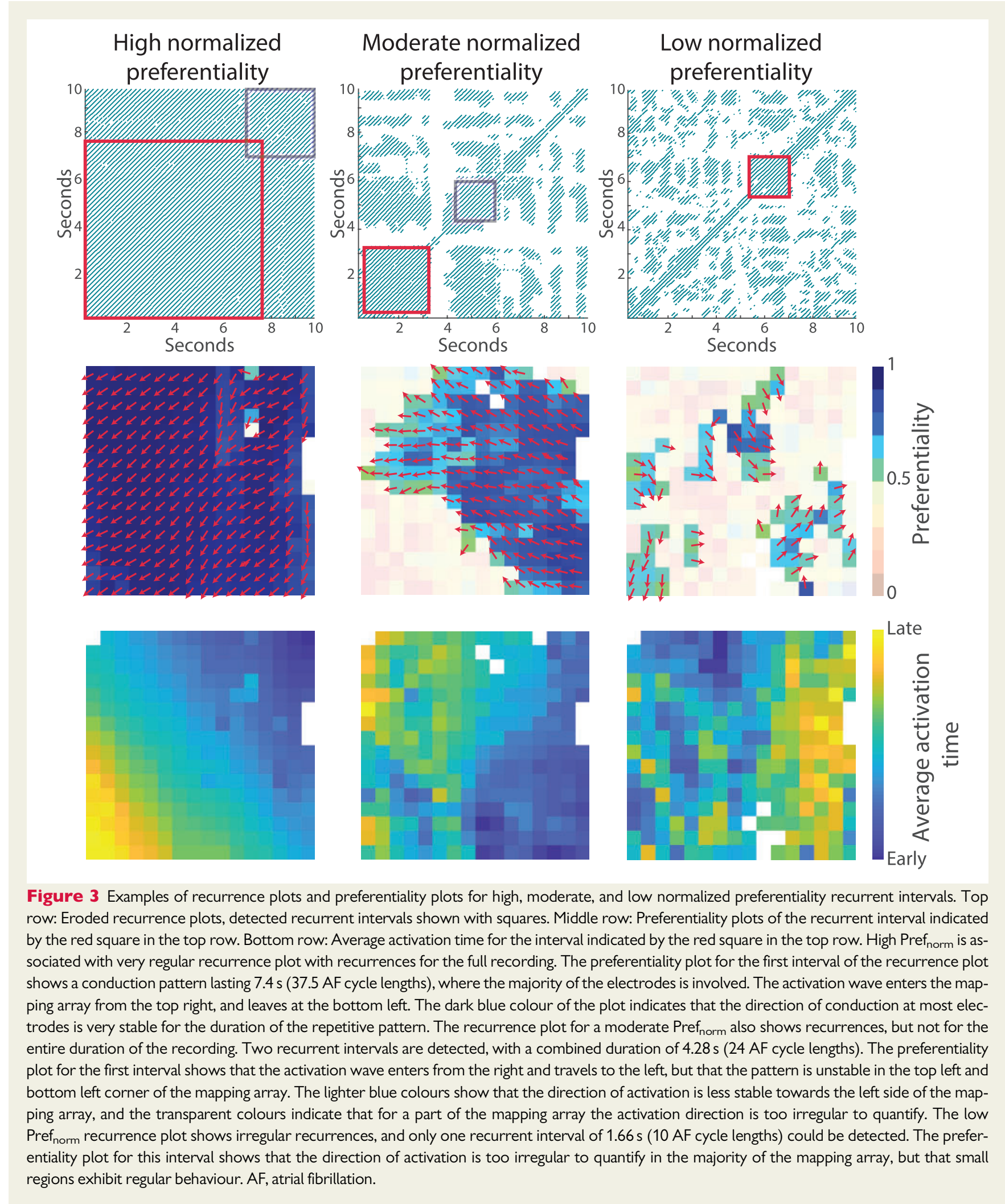

circuit, or conduction away from a source. Re-entry was observed in two patterns (in two patients), a relatively small percentage of all repetitive patterns. When the total re-entry path of the pattern was visible in at least one re-entry cycle, the pattern was classified as re- entry to avoid a negative bias against re-entries. Pivoting waves (waves from one direction pivoting around a line of block and returning to the same direction without crossing the line of block), seen in eight intervals (seven patients), were not counted as re-entries. The 


\section{Paroxysmal AF}

55 intervals

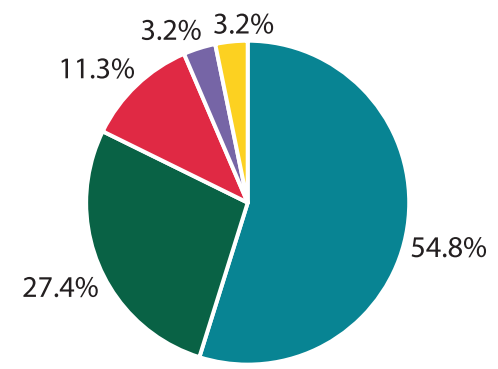

Left atrium

29 intervals

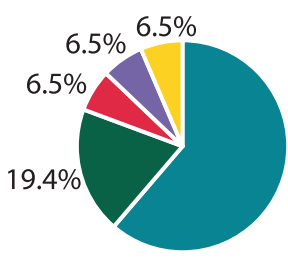

Peripheral with block
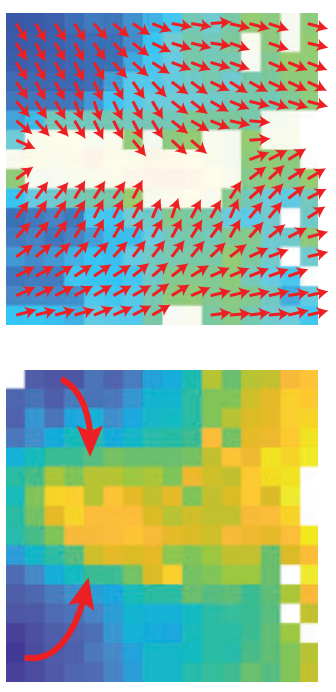

Right atrium 26 intervals

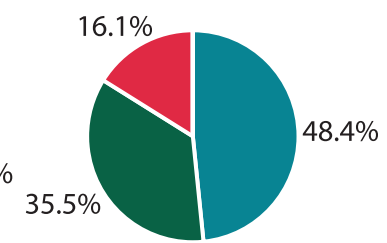

Peripheral without block
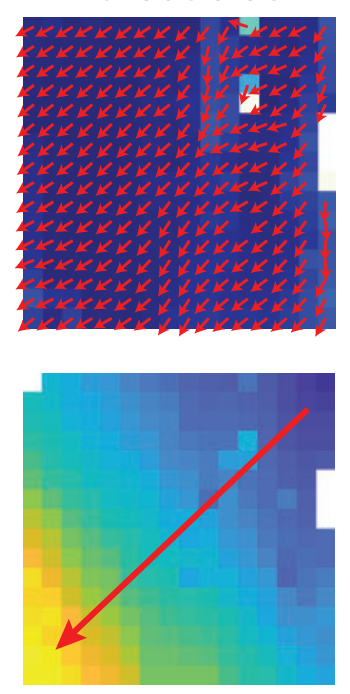

Peripheral with block

- Peripheral without block

Breakthrough

Reentry

Undecided

\section{Persistent AF}

36 intervals

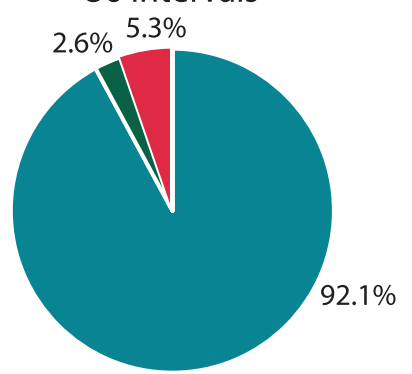

Left atrium

16 intervals

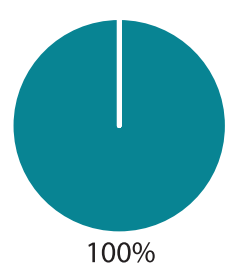

Right atrium

20 intervals

$9.1 \%$

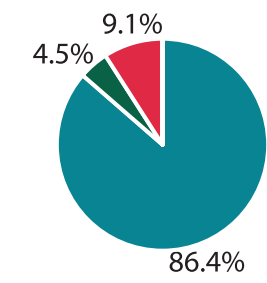

Breakthrough

Reentry
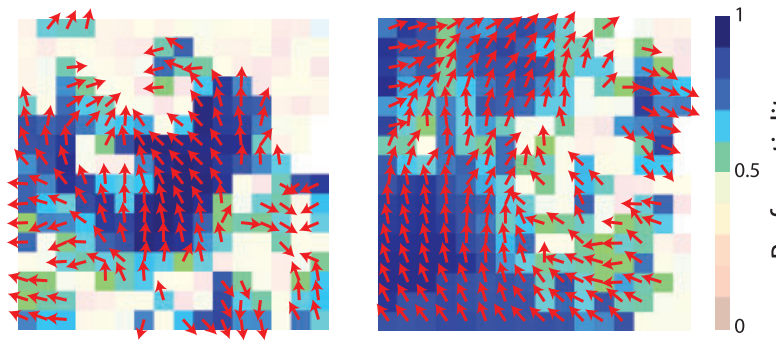

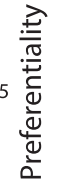
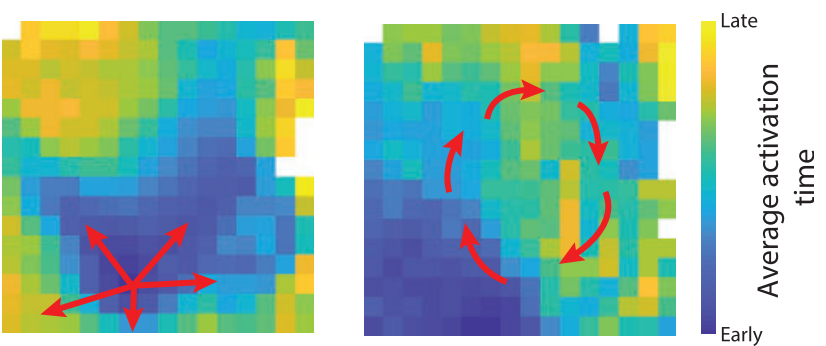

Figure 4 Classification of recurrent intervals in conduction patterns. Top half of the figure shows the occurrence of conduction patterns for paroxysmal and persistent AF. Note that conduction patterns are not mutually exclusive. Bottom half of the figure presents examples of conduction pattern categories. Row three shows the preferentiality map, bottom row shows the average activation time corresponding with the preferentiality map. AF, atrial fibrillation.

low incidence of repetitive re-entry is in line with an earlier analysis of our dataset where incidence of re-entry itself was low. ${ }^{16}$ Hansen et al. found a higher prevalence of re-entry in isolated human atria along endocardial bundles. Their measurements were however performed in the presence of compounds that shorten the AF cycle length which strongly favours re-entry. ${ }^{10}$

\section{Potential applications}

For many years, attempts have been made to design a mapping approach for alternative AF ablation targets. Well-known examples are dominant frequency mapping, ${ }^{23}$ complex fractionated electrogram mapping, ${ }^{24}$ and rotor mapping. ${ }^{25}$ None of these techniques have so far proven to consistently increase success rates of catheter ablation in AF. The inconsistent results from ablation trials may be partly attributable to technological shortcomings of the mapping techniques used. Our earlier work has documented that fractionation indices used in commercially available mapping systems do neither correlate well among each other nor with the incidence of conduction block. ${ }^{26}$ Phase analysis of filtered electrograms as usually used in rotor mapping strongly overestimates rotational activity during AF. ${ }^{16}$ The 
fundamental mechanisms driving AF are still heavily debated by key opinion leaders because of the differences in pre-processing methods, analysis, and interpretation of fibrillation electrograms. ${ }^{9,27}$ Currently proposed hypotheses are that $A F$ is driven or sustained by focal activity emanating from varying locations throughout the atria; ${ }^{9,28}$ spiral wave rotors that may change in complexity during the remodelling process ${ }^{29,30}$ (also suggested by studies using ECG-imaging); ${ }^{31}$ localized stable rotors [showed by using the Focal Impulse and Rotor Modulation (FIRM) approach]; ${ }^{32,33}$ multiple wavelets in the three-dimensional architecture of the atrial wall. ${ }^{20,34}$

In the context of AF ablation beyond PVI, AF sources are particularly interesting when they are stationary or preferentially occur at specific sites. In such cases, conduction close to a source should be highly repetitive and propagating away from this source independently from the actual mechanism of the source. Hence, a repetitive pattern detection technique could help identify and localize AF sources. The existence of repetitive patterns during human AF-as demonstrated by the present study-therefore supports the temporary presence of prevailing conduction paths that may be linked to temporarily active, stationary drivers for AF. Ablation of these drivers could be an opportunity to restore sinus rhythm.

Another potential application of this method is the construction of composite AF conduction maps. The presence of repeating patterns allows combining atrial recordings that have been recorded sequentially into one composite activation map showing these repetitive patterns for the whole atrium. This method is commonly used to produce sinus rhythm activation maps based on several separate recordings throughout the atria. Because of the repetitive nature of activation sequences found in this study, this approach may partly be transferable to mapping of AF. The feasibility of such an approach was demonstrated in the recent RADAR trial, where activation patterns identified in the coronary sinus have been used to link sequential but small activation patterns to one larger composite map. ${ }^{14}$

\section{Limitations}

The mapping arrays used in this study only cover part of the atria. Therefore, we do not know the state of the atria outside of the mapping array. To overcome this limitation, future measurements may use either a larger mapping array or multiple mapping arrays at the same time, or use a single mapping array and a reference signal to create a composite map from sequentially mapped data.

For this study, only $10 \mathrm{~s}$ of data was used for the analysis of recurrent patterns. Patterns that are recurrent with pauses longer than $10 \mathrm{~s}$ between the recurrences would have been missed. Despite our $10 \mathrm{~s}$ recordings, we did find recurrent intervals in the majority of recordings, suggesting that for this type of analysis relatively short recordings could already provide meaningul data.

Preferentiality plots were used to summarize the conduction pattern of individual recurrent intervals. A possible limitation of using preferentiality plots is the compression of temporal information. When a conduction pattern shows regular conduction from left-toright for the first half of the recurrent interval, but then changes to right-to-left conduction for the second half or the recurrent interval, the average conduction direction may be too uncertain to quantify. Visual comparison of the preferentiality plots with activation animations showed that this effect was negligible in this dataset.
The threshold used to decide whether two snapshots are similar was adaptive and based on the expected AF cycle length. This led to a sensitive, but not specific detection of repetitive snapshots. Consequently, not all intervals could be categorized as a conduction pattern, although this occurred in only 2 out of 96 cases.

\section{Conclusion}

High-density epicardial contact mapping in AF patients reveals frequent repetitive conduction patterns. In persistent $A F$ patients, repetitive patterns were less frequent, smaller, and more variable than in paroxysmal AF. The majority of repetitive conduction patterns was (blocked) peripheral waves entering from outside the mapping array. Repetitive breakthrough and re-entry patterns were observed in a small minority of cases. The existence of repetitive patterns during AF suggests the presence of stationary AF drivers. Future research should elucidate whether detection of repetitive patterns can help to locate AF drivers.

\section{Supplementary material}

Supplementary material is available at Europace online.

\section{Funding}

This work was supported by the European Union (ITN PersonalizeAF, Horizon 2020 research and innovation programme PersonalizeAF under the Marie Skłodowska-Curie grant agreement No. 860974 to U.S.); the Netherlands Heart Foundation (CVON2014-09, RACE V: Reappraisal of Atrial Fibrillation: Interaction between hyperCoagulability, Electrical remodelling, and Vascular Destabilization in the Progression of AF to U.S.); and Health Holland, Top Sector Life Sciences \& Health (EXACTIN-AF, PPP allowance LSHM19017 to S.Z.). This paper is part of a supplement supported by an unrestricted grant from the Theo-Rossi di Montelera (TRM) foundation.

Conflict of interest: U.S. received honoraria or consultancy fees from EP Solutions, Roche, and Johnson \& Johnson and research grant from Roche and EP Solutions. U.S. is a shareholder of YourRhythmics BV.

\section{Data availability}

Access to metadata can be granted by the corresponding author based on reasonable request.

\section{References}

1. Hindricks G, Potpara T, Dagres N, Arbelo E, Bax JJ, Blomström-Lundqvist C et al. 2020 ESC guidelines for the diagnosis and management of atrial fibrillation developed in collaboration with the European Association of Cardio-Thoracic Surgery (EACTS). Eur Heart J 2020;1-126. Aug 29:ehaa612. doi: 10.1093/eurheartj/ehaa612

2. January CT, Wann LS, Alpert JS, Calkins H, Cigarroa JE, Cleveland JC et al. AHA ACC/HRS guideline for the management of patients with atrial fibrillation: a report of the American College of cardiology/American heart association task force on practice guidelines and the heart rhythm society. Circulation 2014 Dec 2; 130(23):e199-267.

3. Calkins H, Hindricks G, Cappato R, Kim Y-H, Saad EB, Aguinaga L et al. 2017 HRS/EHRA/ECAS/APHRS/SOLAECE expert consensus statement on catheter and surgical ablation of atrial fibrillation. Europace 2018;20:e1-160.

4. Pooter JD, Strisciuglio T, Haddad ME, Wolf M, Phlips T, Vandekerckhove $Y$ et al. Pulmonary vein reconnection no longer occurs in the majority of patients after a single pulmonary vein isolation procedure. JACC Clin Electrophysiol 2019;5: 295-305. 
5. Verma A, Jiang CY, Betts TR, Chen J, Deisenhofer I, Mantovan R et al. Approaches to catheter ablation for persistent atrial fibrillation. N Engl J Med 2015;372:1812-22.

6. Wong KCK, Paisey JR, Sopher M, Balasubramaniam R, Jones M, Qureshi N et al. No benefit of complex fractionated atrial electrogram ablation in addition to circumferential pulmonary vein ablation and linear ablation: benefit of complex ablation study. Circ Arrhythm Electrophysiol 2015;8:1316-24.

7. Mohanty S, Mohanty P, Trivedi C, Gianni C, Della Rocca DG, Di Biase L, Natale A. Long-term outcome of pulmonary vein isolation with and without focal impulse and rotor modulation mapping. Circ Arrhythmia Electrophysiol 2018;11:1-9.

8. Atienza F, Almendral J, Ormaetxe JM, Moya Á, Martínez-Alday JD, HernándezMadrid A et al. Comparison of radiofrequency catheter ablation of drivers and circumferential pulmonary vein isolation in atrial fibrillation: a noninferiority randomized multicenter RADAR-AF trial. J Am Coll Cardiol 2014;64:2455-67.

9. Lee S, Sahadevan J, Khrestian CM, Cakulev I, Markowitz A, Waldo AL. Simultaneous biatrial high-density (510-512 Electrodes) epicardial mapping of persistent and long-standing persistent atrial fibrillation in patients: new insights into the mechanism of its maintenance. Circulation 2015;132:2108-17.

10. Hansen BJ, Zhao J, Csepe TA, Moore BT, Li N, Jayne LA et al. Atrial fibrillation driven by micro-anatomic intramural re-entry revealed by simultaneous subepicardial and sub-endocardial optical mapping in explanted human hearts. Eur Heart J 2015;36:2390-401.

11. Narayan SM, Shivkumar K, Krummen DE, Miller JM, Rappel W-J. Panoramic electrophysiological mapping but not electrogram morphology identifies stable sources for human atrial fibrillation. Circ Arrhythm Electrophysiol 2013;6:58-67.

12. van Hunnik A, Zeemering S, Podziemski P, Simons J, Gatta G, Hannink L et al. Stationary atrial fibrillation properties in the goat do not entail stable or recurrent conduction patterns. Front Physiol 2018;9:1-16.

13. Maesen B, Zeemering S, Afonso C, Eckstein J, Burton RAB, Hunnik AV et al. Rearrangement of atrial bundle architecture and consequent changes in anisotropy of conduction constitute the 3-dimensional substrate for atrial fibrillation. Circ Arrhythm Electrophysiol 2013;6:967-75.

14. Choudry S, Mansour M, Sundaram S, Nguyen DT, Dukkipati SR, Whang W et al. A multicenter food and drug administration investigational device exemption clinical trial of persistent atrial fibrillation. Circ Arrhythmia Electrophysiol 2020;2-12. Jan;13(1):e007825.

15. Zeemering S, van Hunnik A, van Rosmalen F, Bonizzi P, Scaf B, Delhaas T et al. A novel tool for the identification and characterization of repetitive patterns in high-density contact mapping of atrial fibrillation. Front Physiol 2020 Oct 15;11: 570118. doi:10.3389/fphys.2020.570118.

16. Podziemski P, Zeemering S, Kuklik P, van Hunnik A, Maesen B, Maessen J et al. Rotors detected by phase analysis of filtered, epicardial atrial fibrillation electrograms colocalize with regions of conduction block. Circ Arrhythm Electrophysiol 2018;11:e005858.

17. Zeemering S, Maesen B, Nijs J, Lau DH, Granier M, Verheule S et al. Automated quantification of atrial fibrillation complexity by probabilistic electrogram analysis and fibrillation wave reconstruction. 2012 Annual International Conference of the IEEE Engineering in Medicine and Biology Society IEEE, 2012. pp.6357-60. doi:10.1109/EMBC.2012.6347448.

18. Nattel S, Harada M. Atrial remodeling and atrial fibrillation: recent advances and translational perspectives. J Am Coll Cardiol 2014;63:2335-45.
19. Allessie MA, de Groot MS, Houben RPM, Schotten U, Boersma E, Smeets JL, Crijns $\mathrm{HJ}$ et al. Electropathological substrate of long-standing persistent atrial fibrillation in patients with structural heart disease. Circ Arrhythm Electrophysiol 2010;3:606-15.

20. Eckstein J, Zeemering S, Linz D, Maesen B, Verheule S, Hunnik AV et al. Transmural conduction is the predominant mechanism of breakthrough during atrial fibrillation: evidence from simultaneous endo-epicardial high-density activation mapping. Circ Arrhythm Electrophysiol 2013;6:334-41.

21. de Groon N, van der Does L, Yaksh A, Lanters E, Teuwen C, Knops P et al. Direct proof of endo-epicardial asynchrony of the atrial wall during atrial fibrillation in humans. Circ Arrhythmia Electrophysiol 2016;9:1-7.

22. Aronis KN, Ali RL, Liang JA, Zhou S, Trayanova NA. Understanding AF mechanisms through computational modelling and simulations. Arrhythm Electrophysiol Rev 2019;8:210-9.

23. Lazar S, Dixit S, Marchlinski FE, Callans DJ, Gerstenfeld EP. Presence of left-toright atrial frequency gradient in paroxysmal but not persistent atrial fibrillation in humans. Circulation 2004;110:3181-6.

24. Nademanee K, McKenzie J, Kosar E, Schwab M, Sunsaneewitayakul B, Vasavakul $T$ et al. A new approach for catheter ablation of atrial fibrillation: mapping of the electrophysiologic substrate. J Am Coll Cardiol Elsevier Masson SAS 2004;43 2044-53.

25. Narayan SM, Krummen DE, Rappel W-J. Clinical mapping approach to diagnose electrical rotors and focal. J Cardiovasc Electrophysiol 2012;23:447-54.

26. Lau DH, Maesen B, Zeemering S, Kuklik P, van HA, Lankveld TAR et al. Indices of bipolar complex fractionated atrial electrograms correlate poorly with each other and atrial fibrillation substrate complexity. Hear Rhythm Elsevier 2015;12: 1415-23.

27. Nattel S, Dobrev D. Controversies about atrial fibrillation mechanisms: aiming for order in chaos and whether it matters. Circ Res 2017;120:1396-8.

28. Lee S, Sahadevan J, Khrestian CM, Markowitz A, Waldo AL. Characterization of foci and breakthrough sites during persistent and long-standing persistent atrial fibrillation in patients: studies using high-density (510-512 Electrodes) biatrial epicardial mapping. I Am Heart Assoc 2017;6:1-12.

29. Berenfeld O, Jalife J. Mechanisms of atrial fibrillation. Heart Fail Clin 2016;12 167-78.

30. Jalife J, Berenfeld O, Skanes A, Mandapati R. Mechanisms of atrial fibrillation: mother rotors or multiple daughter wavelets, or both? J Cardiovasc Electrophysiol 1998;9:S2-12.

31. Haissaguerre M, Hocini M, Denis A, Shah AJ, Komatsu $Y$, Yamashita $S$ et al. Driver domains in persistent atrial fibrillation. Circulation 2014;130:530-8.

32. Krummen DE, Baykaner T, Schricker AA, Kowalewski CAB, Swarup V, Miller JM et al. Multicentre safety of adding focal impulse and rotor modulation (FIRM) to conventional ablation for atrial fibrillation. Europace 2017;19:769-74.

33. Narayan SM, Patel J, Mulpuru S, Krummen DE. Focal impulse and rotor modulation ablation of sustaining rotors abruptly terminates persistent atrial fibrillation to sinus rhythm with elimination on follow-up: a video case study. Hear Rhythm Elsevier Inc 2012;9:1436-9.

34. Allessie MA, Groot ND, Houben RPM, Schotten U, Boersma E, Smeets JL et al. Electropathological substrate of long-standing persistent atrial fibrillation in patients with structural heart disease longitudinal dissociation. Circ Arrhythm Electrophysiol 2010;3:606-15. 